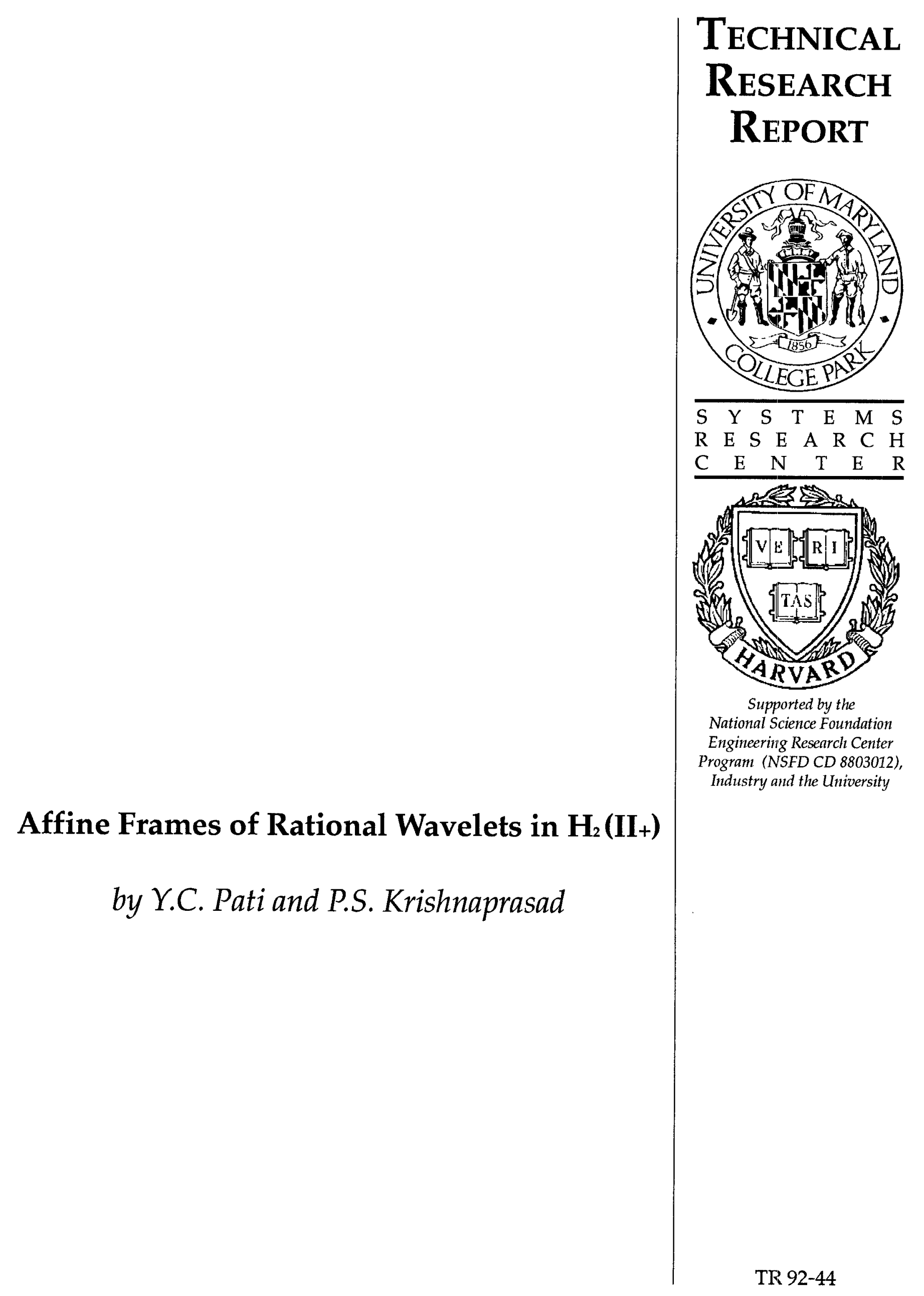




\title{
Affine Frames of Rational Wavelets in $\mathrm{H} 2(\Pi+) *$
}

\author{
Y. C. Pati P. S. Krishnaptasad
}

Electrical Engineering Department and Systems Research Center

University of Maryland, College Park, MD 20742

\begin{abstract}
In this paper we investigate frame decompositions of $\mathrm{H}^{2}\left(\Pi^{+}\right)$as a method of constructing rational approximations to nonrational transfer functions in $\mathrm{H}^{2}\left(\Pi^{+}\right)$. The frames of interest are generated from a single analyzing wavelet. We consider the case in which the analyzing wavelet is rational and show that by appropriate grouping of terms in a wavelet expansion, $\mathrm{H}^{2}\left(\Pi^{+}\right)$can be decomposed as an infinite sum of rational transfer functions which are related to one another by dilation and translation. Criteria for selecting a finite number of terms from such an infinite expansion are developed using time-frequency localization properties of wavelets.
\end{abstract}

*This research was supported in part by the National Sicience Foundation's Engineering Research Centers Program: NSFD CDR 8803012, the Air Force Office of Scientific Research under contract AFOSR-88-0204, and by the AFOSR University Research Initiative Program under grant AFOSR-90. 0105. 



\section{Introduction}

Discrete affine wavelet transforms are a class of transforms defined on function spaces which provide decompositions of functions in terms of dilations and translations of a single function called the analyzing wavelet. In this paper we study wavelet decompositions of the Hardy space $\mathrm{H}^{2}\left(\Pi^{+}\right)$, (where $\Pi^{+}$denotes the half-plane ie $s>0$ ) via rational analyzing wavelets. A rational wavelet in $\mathrm{H}^{2}\left(\mathrm{II}^{+}\right)$can of course be viewed as the transfer function of a finite-dimensional causal linear time invariant system. We show that any function in $\mathrm{H}^{2}\left(\mathrm{II}^{+}\right)$can be written as an infinite sum of real-rational functions where the terms in the summation are related to one another by translations and dilations. We refer to such a decomposition as a Wavelet System (WS) decomposition of $\mathrm{H}^{2}\left(\Pi^{+}\right)$ . In requiring rationality of the analyzing wavelet, orthogonality of the 'basis' functions is sacrificed. However, we show that frames for $\mathrm{II}^{2}\left(\Pi^{+}\right)$can be constructed from dilates and translates of rational analyzing wavelets. The frame property assures completeness and computability of the decomposition. In problems involving dynamical systems with nonrational transfer functions (i.e. infinite-dimensional systems), one is often required to approximate the system by a simpler (finitc-dimensional) system. Equivalently, one is required to contruct rational approximations to nonrational transfer functions. We consider here the problem of constructing a causal rational approximation to a causal nonrational transfer function by selecting a finite number of terms from the WS expansion. Translations and dilations of the analyzing wavelet generate a family of functions which are well localized in the time-frequency plane. We discuss criteria for selection of terms in a manner which exploits time-frequency concentrations of affine wavelets and of the original (nonrational) transfer function. Possible applications of the methods described in this paper are in system identification and compensator design.

Central to the theme of this paper is the action of the affine group Aff(1) on transfer functions. It is well-known that the affine group Aff $(1)$ acts on transfer functions in several ways. These were investigated in some depth in [2]. Two such actions are given by (for $\alpha>0, \beta \in \mathbb{R}$ ),

$$
\begin{aligned}
\text { (a) } g(s) & \longrightarrow g(\alpha s+\beta) \\
\text { and } \quad(\mathrm{b}) \quad g(s) & \longrightarrow \frac{\alpha g(s)}{1+\alpha \beta \log (s)}
\end{aligned}
$$

The first action can be imbedded in an action of the group $S 1(2)$ on transfer functions due to Brockett. Some related group theoretical aspects are investigated in the paper by Grossmann and Morlet [13]. This paper is closest in spirit to our work. The key difference is that Grossman and Morlet focus on continuous wavelet transforms and nonrational wavelets.

\section{Discrete Wavelet Transforms and Frames for Hilbert Spaces}

Wavelet transforms provide a means for decomposition of function spaces via dilates and translates of a single function known as the anclyzing wavelet (also referred to as 
the mother wavelet). Although wavelet transforms have been studied in the context of a number of different function spaces, a great deal of attention has been devoted to wavelet transforms on $\mathrm{L}^{2}(\mathbb{R})$. The contents of this paper rely primarily on results derived for $\mathrm{L}^{2}(\mathrm{IR})$. Define, for $a_{0}>0$, the dilation operator $D_{a}$ on $\mathrm{L}^{2}(\mathrm{IR})$ by $D_{a} f(x)=a^{1 / 2} f(a x)$ and the translation operator $T_{b}$ on $\mathrm{L}^{2}(\mathrm{IR})$ by $T_{b} f(x)=f(x-b)$. Discrete affine wavelet transforms on $L^{2}(\mathbb{R})$ allow representation of any $f \in L^{2}(\mathbb{R})$ as

$$
f=\sum_{m \in \mathbf{Z}} \sum_{n \in \mathbf{Z}} c_{m, n} u_{m, n},
$$

where $\psi$ is the analyzing wavelet and,

$$
\psi_{m, n}(x)=T_{n b_{0}} D_{a_{0}^{m}} \psi(x)=a_{0}^{m / 2} \psi\left(a_{0}^{m} x-n b_{0}\right)
$$

for fixed constants $a_{0}>0$ and $b_{0}$.

It has been shown (see e.g. [5]) that it is possible to find functions $\psi$ and constants $a_{0}$ and $b_{0}$ for which the sequence $\left\{\psi_{m, n}\right\}_{m, n \in \mathbf{Z}}$ forms an orthonormal basis for $L^{2}(\mathbb{R})$. In this case, (1) is the usual orthonormal basis expansion of a function. i.e. $c_{m, n}=\left\langle f, \psi_{m, n}\right\rangle$. However, the requirement of orthogonality of translates and dilates severely restricts allowable choices for the analyzing wavelet. A great deal of freedom in the choice of analyzing wavelets can be purchased at the cost of orthogonality if one were to consider frames instead.

\subsection{Frames}

Frames, which were first introduced by Duffin and Schaeffer in [7], are natural generalizations of orthonormal bases for Hilbert spaces.

Definition 2.1 Given a Hilbert space $\mathcal{H}$ and a sequence of vectors $\left\{h_{n}\right\}_{n=-\infty}^{\infty} \subset \mathcal{H}$, $\left\{h_{n}\right\}_{n=-\infty}^{\infty}$ is called a frame if there exist constants $A>0$ and $B<\infty$ such that

$$
A\|f\|^{2} \leq \sum_{n}\left|<f, h_{n}>\right|^{2} \leq B\|f\|^{2},
$$

for every $f \in \mathcal{H}$. $A$ and $B$ are called the frame bounds.

Definition 2.2 Given a frame $\left\{h_{n}\right\}$ in a Hilbert space $\mathcal{H}$, with frame bounds $A$ and $B$, we can define the frame operator $S: \mathcal{H} \rightarrow \mathcal{H}$ as follows. For any $f \in \mathcal{H}$,

$$
S f=\sum_{n}<f, h_{n}>h_{n}
$$

The following theorem lists some properties of the frame operator. Proof of these and other related properties of frames can be found in [14] or [6].

\section{Theorem 2.1}

(1) $S$ is a bounded linear operator with $A I \leq S \leq B I$, where $I$ is the identity operator in $\mathcal{H}$. 
(2) $S$ is an invertible operator with $B^{-1} I \leq S^{-1} \leq A^{-1} I$.

(3) Since $A I \leq S \leq B I$ implies that $\left\|I-\frac{2}{A+B} S\right\| \leq 1, S^{-1}$ can be computcd via the Neumann series,

$$
S^{-1}=\frac{2}{A+B} \sum_{k=0}^{\infty}\left(I-\frac{2}{A+B} S\right)^{k} .
$$

(4) The sequence $\left\{S^{-1} h_{n}\right\}$ is also a frame, called the dual frame, with frame bounds $B^{-1}$ and $A^{-1}$.

(5) Given any $f \in \mathcal{H}, f$ can be written in terms of frame elements as

$$
f=\sum<f, S^{-1} h_{n}>h_{n}=\sum<f, h_{n}>S^{-1} h_{n} .
$$

(6) If there exists another sequence of coefficients $\left\{a_{n}\right\}$ such that $f=\sum a_{n} h_{n}$,

$$
\sum\left|a_{n}\right|^{2}=\sum\left|<f, S^{-1} h_{n}>\right|^{2}+\sum\left|<f, S^{-1} h_{n}>-a_{n}\right|^{2} .
$$

\subsection{Frames of Affine Wavelets}

Given a function $\psi$ in $L^{2}(\mathrm{IR})$ satisfying the admissibility condition

$$
\int_{\mathbb{R}} \frac{|\widehat{\psi}(\omega)|^{2}}{|\omega|} d \omega<\infty
$$

where $\widehat{\psi}$ is the Fourier transform of $\psi$, under mild additional hypothesis, it is possible to choose parameters $a_{0}$ and $b_{0}$ such that $\left(\psi, a_{0}, b_{0}\right)$ generates an affine frame for $\mathrm{L}^{2}(\mathbb{R})$, i.e. the sequence $\left\{\psi_{m, n}\right\}$ as defined by (2) forms a frame for $\mathrm{L}^{2}(\mathrm{IR})$. Numerically, $a_{0}$ and $b_{0}$ can be determined by application of a theorem of Daubechies [6] (see also [17]).

\subsubsection{Time-Frequency Localization in Affine Wavelets}

The key property of affine wavelet decompositions which has demonstrated noted benefits in a number of applications, is the time-frequency localization which arises due to the translations and dilations. The concepts of time and frequency concentrations of a function can be precisely defined (c.f. [17]), but roughly speaking these are intervals in the time or frequency domains which contain 'most' of the energy in the signal. Let $\Omega(\psi)=\left[\omega_{0}(\psi), \omega_{1}(\psi)\right]$ denote the frequency concentration of $\psi$ and $R(\psi)=\left[t_{0}(\psi), t_{1}(\psi)\right]$ $\left(t_{0} \geq 0\right)$ denote the time concentration of $\psi$, where $\psi$ is an admissible analyzing wavelet. Then $\psi$ is a function which is concentrated in the time frequency plane on the rectangle $\mathcal{Q}=\Omega(\psi) \times R(\psi)$ and each of the wavelets $\psi_{m, n}$, are concentrated on rectangles

$$
\mathcal{Q}_{m, n}=\left[a_{0}^{-m}\left(t_{0}(\psi)+n b_{0}\right), a_{0}^{-m}\left(t_{1}(\psi)+n b_{0}\right)\right] \times\left[a_{0}^{m} \omega_{0}(\psi), a^{m} \omega_{1}(\psi)\right] .
$$

(Assuming symmetry about $\omega=0$, only positive frequencies are considered.) 


\section{Wavelet Decompositions of $\mathrm{H}^{2}(\Pi+)$}

\subsection{The Hardy Space $\mathrm{H}^{2}\left(\Pi^{+}\right)$}

The class of transfer functions of interest to us is the Hardy space $\mathrm{H}^{2}\left(\Pi^{+}\right)$where $\Pi+$ denotes, the half-plane $\Re e s>0$. For completeness, a few important properties of $\mathrm{H}^{2}\left(\Pi^{+}\right)$are reviewed here. For further discussion on $\mathrm{H}^{p}$ spaces see $[15,8,9]$. In particular [15] discusses the space $H^{p}\left(\Pi^{+}\right)$and the relationship between $H^{p}$ spaces on the unit disc and $\mathrm{H}^{p}$ spaces of $\Pi+$

Definition 3.1 Given a function $F$ which is analytic in $\Pi^{+}, F$ is said to belong to the class $\mathrm{H}^{2}\left(\Pi^{+}\right)$if

$$
\sup _{x>0} \int_{-\infty}^{\infty}|F(x+i y)|^{2} d y<\infty .
$$

$\mathrm{H}^{2}\left(\Pi^{+}\right)$is a Banach space with norm (denoted $\|\cdot\|_{H^{2}}$ ) defined by (8).

Some of the most basic properties of $\mathrm{H}^{2}\left(\Pi^{+}\right)$are captured by the following theorem (c.f. [15]).

Theorem 3.1 Given $F \in \mathrm{H}^{2}\left(\Pi^{+}\right)$, the following are truc:

(1) The nontangential limit of $F$ exists at almost every point on the imaginary axis.

(2) The boundary value function of $F$ is in $\mathrm{L}^{2}(\mathrm{R})$ and,

$$
F(x+i y)=\frac{1}{\pi} \int_{\mathbb{R}} F(i \omega) \frac{x}{x^{2}+(y-\omega)^{2}} d \omega, \quad x>0
$$

(3) The functions $F_{x}(y)=F(x+i y)$ converge in $L^{2}$ norm to $F(i y)$ as $x \rightarrow 0$.

The elements of $\mathrm{H}^{2}\left(\Pi^{+}\right)$are transfer functions of causal input-output stable linear systems. More precisely, we have the classical result,

Theorem 3.2 (Paley-Wiener) A complex-valued function $F$ is in $\mathrm{H}^{2}\left(\Pi^{+}\right)$if and only if,

$$
F(s)=\int_{0}^{\infty} f(t) e^{-s t} d t,
$$

for some $f \in \mathrm{L}^{2}(0, \infty)$. Furthermore this representation is unique.

By the Paley-Wiener theorem,

$$
\frac{1}{2 \pi} \int_{\mathbb{R}} F(i \omega) e^{i \omega t} d \omega=0 \text { for } t<0 .
$$

Hence boundary values of functions in $\mathrm{H}^{2}\left(\Pi^{+}\right)$comprise a subspace $\mathcal{D}$ of $\mathrm{L}^{2}(\mathbb{R})$ characterized by $(9)$. We also know by Theorem 3.1 that given the boundary value function $F(i \omega), F$ can be recovered on the right half-plane by the Poisson integral. Also $\|F\|_{H^{2}}=\|\widetilde{F}\|_{L^{2}}$ where $\widetilde{F}$ is the nontangential limit of $F$.

A Hilbert space structure is placed on $\mathrm{H}^{2}\left(\Pi^{+}\right)$by the inner product defined by,

$$
\langle F, G\rangle_{H^{2}}=\int_{-\infty}^{\infty} F(i \omega) \overline{G(i \omega)} d \omega .
$$


Remark: In what follows, we take the liberty of identifying $\mathrm{I}^{2}\left(\Pi^{+}\right)$with $\mathcal{D}$. We use $\omega$ to denote the real frequency variable and write $F(\omega)$ to denote the boundary value function $F(i \omega)$. The complex frequency variable will be denoted by $s$. In the remainder of this paper, we adopt the following notation:

$\mathrm{RH}^{2}\left(\mathrm{II}^{+}\right)$: Real-rational functions in $\mathrm{H}^{2}\left(\mathrm{II}^{+}\right)$.

$\mathrm{H}_{\mathbb{R}}^{2}\left(\Pi^{+}\right)$: Functions in $\mathrm{H}^{2}\left(\Pi^{+}\right)$which are Laplace transtorms of real-valued functions in $\mathrm{L}^{2}(0, \infty)$.

Note that $\mathrm{RH}^{2}\left(\Pi^{+}\right) \subset \mathrm{H}_{\mathbb{R}}^{2}\left(\Pi^{+}\right)$.

\subsection{Wavelet Transforms on $\mathrm{H}^{2}(\Pi+)$}

By the Paley-Wiener theorem $\mathrm{RH}^{2}(\mathrm{II}+)$ represents transfer functions of causal finitedimensional linear systems with square integrable weighting patterns. Of particular interest to us is the case where the analyzing wavelet $\Psi$ belongs to the class $\mathrm{RH}^{2}\left(\Pi^{+}\right)$. by,

For any $F \in \mathrm{H}^{2}\left(\Pi^{+}\right)$define the restriction of $F$ to vertical lines in the right half-plane

$$
F_{x}(y)=F(x+i y), \quad x>0 .
$$

Also define the Fourier transform along vertical lines in $\Pi+b y$,

$$
\widehat{F}_{x}(u)=\frac{1}{2 \pi} \int_{\mathbb{R}} F(x+i y) e^{i u y} d y
$$

for $F \in \mathrm{H}^{2}\left(\Pi^{+}\right)$.

Now let $\Psi \in \mathrm{RH}^{2}\left(\Pi^{+}\right) \subset \mathrm{H}^{2}\left(\Pi^{+}\right)$be our candidate for an analyzing wavelet, and suppose that for any $x>0 \Psi$ satisfies the admissibility condition,

$$
C_{\Psi_{x}}=\int_{\mathbb{R}} \frac{\left|\widehat{\Psi}_{x}(u)\right|^{2}}{|u|} d u<\infty, \quad x>0 .
$$

Under these assumptions, a family of continuous wavelet transforms can be defined on $\mathrm{H}^{2}\left(\Pi^{+}\right)$. Let,

$$
\Psi^{(a, b)}(s)=|a|^{1 / 2} \Psi(a s-i b), \quad a, b \in \mathbb{R}, \quad a>0 .
$$

Then for any $F \in \mathrm{H}^{2}\left(\Pi^{+}\right)$define the continuous wavelet transform on the line $\Re e s=x$ by,

$$
W_{x} F(a, b)=\int_{\mathbb{R}} F_{x}(y) \overline{\Psi_{x}^{(a, b)}(y)} d y .
$$

Inversion of this transform is accomplished by the inversion formula,

$$
F_{x}(y)=\frac{1}{C_{\Psi_{x}}} \int_{\mathbb{R}} \int_{\mathbb{R}} W_{x} F(a, b) \Psi_{x}^{(a, b)}(y) \frac{d a d b}{a^{2}} .
$$

To define a discrete wavelet transform with respect to $\Psi$, let

$$
\Psi_{m, n}(s)=a_{0}^{m / 2} \Psi\left(a_{0}^{m} s-i n b_{0}\right) \quad a_{0}>0 . \quad \text { 凡e } s=x .
$$


Assume $\Psi$ satisfies the admissibility condition and let $a_{0}>0$ and $b_{0}$ be such that the family $\left\{\Psi_{m, n}\right\}_{m, n \in \mathbf{Z}}$, is a frame for $\mathcal{D}$ which is a closed subspace of $L^{2}(\mathrm{IR})$. Thus for any $F \in \mathrm{H}^{2}\left(\Pi^{+}\right)$we have that

$$
F_{x}(y)=\sum_{m} \sum_{n}\left\langle F, S^{-1} \Psi_{m, n}\right\rangle \Psi_{m, n}
$$

where $S$ is the frame operator associated with the frame $\left\{\Psi_{m, n}\right\}$.

\section{Wavelet System Transfer Functions}

In this paper we restrict discussion to affine frames constructed on the imaginary axis. Hence we require admissibility of the analyzing wavelet $\Psi(s)$ for fie $s=0$ and consider the boundary values of $\mathrm{H}^{2}\left(\Pi^{+}\right)$.

Let $\Psi(\omega)$ denote the nontangential limit of an admissible analyzing wavelet $\Psi(s)$ and let $\left(a_{0}, b_{0}\right)$ be such that $\left(\Psi, a_{0}, b_{0}\right)$ generates an affine frame for $\mathrm{H}^{2}\left(\Pi^{+}\right)$. Then any $F \in \mathrm{H}^{2}\left(\Pi^{+}\right)$can be represented as

$$
F(\omega)=\sum_{m} \sum_{n} c_{m, n} \Psi_{m, n}(\omega)
$$

where as before $\Psi_{m, n}(\omega)=a_{0}^{m / 2} \Psi\left(a_{0}^{m} \omega-n b_{0}\right)$. Assuming that $F \in \mathrm{H}^{2}\left(\Pi^{+}\right)$is the Laplace transform of a real-valued weighting pattern in $\mathrm{L}^{2}(0, \infty)$, and $\Psi$ is a real-rational wavelet, we can ask the question; is an arbitrary truncation of the frame expansion (12) the transfer function of a real weighting pattern? Since $\mathrm{RH}^{2}\left(\Pi^{+}\right)$arises as the image under the Laplace transform of certain real-valued weighting patterns, if the analyzing wavelet $\Psi \in \mathrm{RH}^{2}\left(\Pi^{+}\right)$then $\Psi_{m, n}(-\omega)=\Psi_{m, n}^{*}(\omega)$ for $n=0$ and $m \in \mathbb{Z}$. However, this symmetry is violated for $n \neq 0$. To circumvent this problem, we can group together positive and negative translates of the analyzing wavelet to form what we call a wavelet system transfer function.

Definition 4.1 Given $\Psi \in \mathrm{RH}^{2}\left(\Pi^{+}\right) \subset \mathrm{H}^{2}\left(\Pi^{+}\right)$a wavelet system transfer function is defined as a function of the form,

$$
G^{m, n}(s)=\alpha \Psi_{m, n}(s)+\bar{\alpha} \Psi_{m,-n}(s)
$$

where $\bar{\alpha}$ denotes the complex conjugate of $\alpha$.

Proposition 4.1 Let $F(s)$ be a real-rational function in $\mathrm{H}^{2}\left(\Pi^{+}\right)$. Then, the wavelet system transfer function defined by

$$
G^{m, n}(s)=\alpha F_{m, n}(s)+\bar{\alpha} F_{m,-n}(s)
$$

is also a real-rational function in $\mathrm{H}^{2}\left(\Pi^{+}\right)$.

Proof: Let $F(\omega)$ and $G^{m, n}(\omega)$ denote the nontangential limits of $F(s)$ and $G^{m, n}(s)$ respectively. Since $\mathrm{H}^{2}\left(\Pi^{+}\right)$is a closed linear space, it is clear that $G^{m, n}(s) \in \mathrm{H}^{2}\left(\Pi^{+}\right)$. It 
is also clear that $G^{m, n}(s)$ is rational. To show that $G^{m, n}(s)$ is real-rational we only need to show that

$$
G^{m, n}(-\omega)={\overline{G^{\prime}}}^{m, n}(\omega)
$$

Now,

$$
\begin{aligned}
G^{m, n}(-\omega) & =\alpha F_{m, n}(-\omega)+\bar{\alpha} F_{m,-n}(-\omega) \\
& =\alpha a_{0}^{m / 2} F\left(-a_{0}^{m} \omega-n b_{0}\right)+\bar{\alpha} a_{0}^{m / 2} F\left(-a_{0}^{m} \omega+n b_{0}\right) \\
& =\alpha a_{0}^{m / 2} F\left(-\left(a_{0}^{m} \omega+n b_{0}\right)\right)+\bar{\alpha} a_{0}^{m / 2} F\left(-\left(a_{0}^{m} \omega-n b_{0}\right)\right) \\
& =\alpha a_{0}^{m / 2} \bar{F}\left(a_{0}^{m} \omega+n b_{0}\right)+\bar{\alpha} a_{0}^{m / 2} \bar{F}\left(a_{0}^{m} \omega-n b_{0}\right) \\
& =\alpha \bar{F}_{m,-n}(\omega)+\bar{\alpha} \bar{F}_{m, n}(\omega) \\
& =\bar{G}^{m, n}(\omega) .
\end{aligned}
$$

Grouping together translates of the analyzing wavelet to form WS transfer functions as in Definition 4.1, requires that the coefficients that appear in front of each of the two individual wavelet terms be complex conjugates of one another. For WS transfer functions to be useful, we need to verify that the coefficients $\left\langle F, S^{-1} \Psi_{m, n}\right\rangle$ satisfy this condition for a interesting class of functions in $\mathrm{H}^{2}\left(\Pi^{+}\right)$. In verifying this condition, we make use of the following proposition.

Proposition 4.2 Let $f$ and $g$ be boundary values of functions in $\mathrm{H}^{2}\left(\Pi^{+}\right)$. Also let $f$ and $g$ be such that $f(-\omega)=\overline{f(\omega)}$, and $g(-\omega)=\overline{g(\omega)}$. then,

$$
\left\langle f, g_{m, n}\right\rangle=\overline{\left\langle f, g_{m,-n}\right\rangle} \text {. }
$$

Proof: (See Appendix A)

Using Proposition 4.2, we can show that for functions in $\mathrm{H}^{2}\left(\Pi^{+}\right)$, which are the Laplace transforms of real-valued functions in $\mathrm{L}^{2}(0, \infty)$, the wavelet coefficients do indeed satisfy the conjugation condition required for utility of the concept of WS transfer functions.

Lemma 4.1 Let $f$ and $g$ be boundary values of functions in $\mathrm{H}^{2}\left(\Pi^{+}\right)$such that $f(-\omega)=$ $\overline{f(\omega)}$, and $g(-\omega)=\overline{g(\omega)}$. Let $a_{0}>0$ and $b_{0}$ be such that $\left\{g_{m, n}\right\}_{m, n \in \mathbf{Z}}$ is a frame and let $S$ be the associated frame operator. Then,

$$
c_{m, n}=\left\langle f, S^{-1} g_{m, n}\right\rangle=\overline{\left\langle f, S^{-1} g_{m,-n}\right\rangle}=\overline{c_{m,-n}} .
$$

Proof: Since the frame operator $S$ is self-adjoint,

$$
\left\langle f, S^{-1} g_{m, n}\right\rangle=\left\langle S^{-1} f, g_{m, n}\right\rangle \text {. }
$$

Therefore by Proposition 4.2 we need only check that if $f(-\omega)=\overline{f(\omega)}$ then $S^{-1} f(-\omega)=$ $\overline{S^{-1} f(\omega)}$. Now,

$$
\left(S^{-1} f\right)(-\omega)=\frac{2}{A+B} \sum_{k=0}^{\infty}\left(I-\frac{2}{A+B} S\right)^{k} f(-\omega)
$$


Let

$$
\begin{aligned}
& G^{m, n}(\omega)=\left\langle f, g_{m, n}\right\rangle g_{m, n}(\omega)+\left\langle f, g_{m,-n}\right\rangle g_{m,-n}(\omega) \quad m \in \mathbb{Z}, n \in \mathbb{Z}^{+}, n \neq 0 \\
& G^{m, 0}(\omega)=\left\langle f, g_{m, 0}\right\rangle g_{m, 0}(\omega) \quad m \in \mathbb{Z} .
\end{aligned}
$$

Then

$$
\begin{aligned}
(S f)(-\omega) & =\sum_{m}\left[G^{m, 0}(-\omega)+\sum_{n=1}^{\infty} G^{m, n}(-\omega)\right] \\
& =\sum_{m}\left[\overline{G^{m, 0}}(\omega)+\sum_{n=1}^{\infty} \overline{G^{m, n}}(\omega)\right] \\
& =\overline{(S f)}(\omega) .
\end{aligned}
$$

Thus,

$$
\left[\left(I-\frac{2}{A+B} S\right) f\right](-\omega)=\overline{\left(I-\frac{2}{A+B} S\right) f}(\omega)
$$

and together with $(13)$, we get $\left(S^{-1} f\right)(-\omega)=\overline{\left(S^{-1} f\right)}(\omega)$, which completes the proof.

The above observations lead us to the definition of what we will call the Wavelet System Transform on $\mathrm{H}^{2}\left(\Pi^{+}\right)$. We first define the space containing the image of the WS transform operator.

Definition 4.2 Define $\mathcal{R}(J)$ as the space of sequences of functions $\left\{F_{k}\right\}_{k \in \mathcal{T}}$ such that $F_{k} \in \mathrm{RH}^{2}\left(\Pi^{+}\right) \subset \mathrm{H}^{2}\left(\Pi^{+}\right)$for all $k \in \mathcal{J}$ and

$$
\left\|\sum_{k \in \mathcal{J}} F_{k}\right\|_{H^{2}}^{2}<\infty .
$$

Definition 4.3 Let $\Psi \in \mathrm{H}^{2}\left(\Pi^{+}\right)$be an admissible real-rational analyzing wavelet and $a_{0}>0, b_{0}$ such that $\left(\Psi, a_{0}, b_{0}\right)$ generates an affine frame for $\mathrm{H}^{2}\left(\Pi^{+}\right)$. The Wavelet System transform is defined by the operator $H_{\Psi}: \mathrm{H}_{\mathbb{R}}^{2}(\Pi+) \longrightarrow \mathcal{R}\left(\mathbb{Z} \times \mathbb{Z}^{+}\right)$,

$$
H_{\Psi} F=\left\{F^{m, n}\right\}_{m \in \mathbf{Z}, n \in \mathbf{Z}^{+}},
$$

where for any $F \in \mathrm{H}^{2}\left(\Pi^{+}\right), F^{m, n}$ is given by,

$$
\begin{aligned}
& F^{m, n}(\omega)=\left\langle F, S^{-1} \Psi_{m, n}\right\rangle \Psi_{m, n}(\omega)+\left\langle F, S^{-1} \Psi_{m,-n}\right\rangle \Psi_{m,-n}(\omega) \quad m \in \mathbb{Z}, n \in \mathbb{Z}^{+}, \quad n \neq 0 \\
& F^{m, 0}(\omega)=\left\langle F, S^{-1} \Psi_{m, 0}\right\rangle \Psi_{m, 0}(\omega) \quad m \in \mathbb{Z} .
\end{aligned}
$$

The operator $H_{\Psi}$ defines an invertible isometry from $\mathrm{H}^{2}\left(\Pi^{+}\right)$to $\mathcal{R}\left(\mathbb{Z} \times \mathbb{Z}^{+}\right)$, (where (14) defines the norm on $\mathcal{R}(\mathcal{J})$ ). Inversion of $H_{\bar{w}}$ involves a simple summation of terms in the sequence $\left\{F^{m, n}\right\}$.

Putting together Proposition 4.1 and Lemma 4.1, we can now state the main decomposition theorem for $\mathrm{H}_{\mathbb{R}}^{2}\left(\Pi^{+}\right)$. 
Theorem 4.1 Let $\Psi \in \mathrm{RH}^{2}\left(\Pi^{+}\right)$be an admissible analyzing wavelet, and $a_{0}>0, b_{0}$ such that $\left(\Psi, a_{0}, b_{0}\right)$ generates an affine frame for $\mathrm{H}^{2}\left(\Pi^{+}\right)$. Then, any $F$ in $\mathrm{H}_{\mathbb{R}}^{2}\left(\Pi^{+}\right)$ can be represented as,

$$
F=\sum_{m} \sum_{n=0}^{\infty} F^{m, n},
$$

where, each $F^{m, n}\left(\in \mathrm{RH}^{2}\left(\Pi^{+}\right)\right)$is a wavelet system transfer function defined by,

$$
\begin{aligned}
& F^{m, n}=\left\langle F, S^{-1} \Psi_{m, n}\right\rangle \Psi_{m, n}+\overline{\left\langle F, S^{-1} \Psi_{m, n}\right\rangle} \Psi_{m,-n} \quad m \in \mathbb{Z}, \quad n=1,2, \ldots \\
& F^{m, 0}=\left\langle F, S^{-1} \Psi_{m, 0}\right\rangle \Psi_{m, 0} \quad m \in \mathbb{Z} .
\end{aligned}
$$

Note that if the analyzing wavelet $\Psi$ is of degree $N$, then the degree of each $F^{m, n}$ is bounded by $2 N$.

\subsection{Example: Rational Analyzing Wavelet for $\mathrm{H}^{2}(\Pi+)$}

As an example of a rational analyzing wavelet for $\mathrm{H}^{2}\left(\Pi^{+}\right)$, consider the function

$$
\Psi(s)=\frac{1}{(s+\gamma)^{2}+\xi^{2}}, \quad \gamma, \xi>0 .
$$

It is easily verified that $\Psi(s)$ is in $\mathrm{H}^{2}\left(\Pi^{+}\right)$and furthermore $\Psi$ is an admissible analyzing wavelet i.e.,

$$
\int_{\mathbb{R}} \Psi(x+i y) d y=0 \quad \text { for } \quad x>0 .
$$

Taking the inverse Laplace transform of $\Psi$, the weighting pattern $\psi(t)$ corresponding to $\Psi$ is given by,

$$
\psi(t)= \begin{cases}\xi^{-1} e^{-\gamma t} \sin (\xi t) & \text { for } t \geq 0 \\ 0 & \text { for } t<0\end{cases}
$$

The $\mathrm{H}^{2}\left(\Pi^{+}\right)$norm of $\Psi$ can also be found to be,

$$
\|\Psi\|_{H^{2}}^{2}=\frac{2 \pi}{4 \xi^{2}}\left(\frac{1}{\gamma}-\frac{\cos (\arctan (\xi / \gamma))}{\sqrt{\xi^{2}+\gamma^{2}}}\right)
$$

Figure 1 and 2 show this wavelet $\Psi$ evaluated on the imaginary axis, and the corresponding weighting pattern $\psi$ for different values of $\xi$ and $\gamma$.

\subsection{Constructing an Affine Frame for $\mathrm{H}^{2}\left(\Pi^{+}\right)$from $\Psi$}

For the purpose of numerically determining values of the dilation stepsize $a_{0}$ and translation stepsize $b_{0}$ such that $\left(\Psi, a_{0}, b_{0}\right)$ generates an affine frame for $\mathrm{H}^{2}\left(\Pi^{+}\right)$we can utilize Theorem C.1 and Corollary C.1 in Appendix C. Figures 3-4 show the results of applying Theorem C.1 and Corollary C.1 for the case where $a_{0}=2.0$. Hence for $a_{0}=2.0$ and $0<b_{0} \leq 17$ the sequence $\left\{\Psi_{m, n}\right\}$ forms an affine frame for $\mathrm{H}^{2}\left(\Pi^{+}\right)$. 

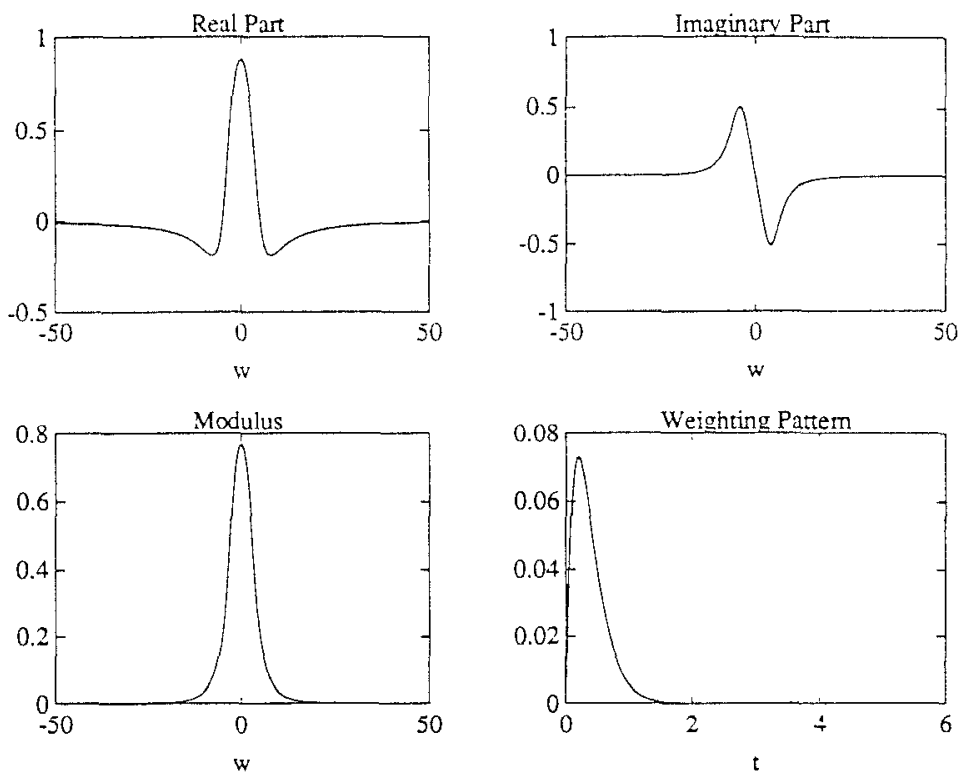

Figure 1: $\mathrm{H}^{2}\left(\Pi^{+}\right)$wavelet $\Psi$ for $\gamma=5, \xi=1$, and weighting pattern $\psi$.

\section{Properties of Wavelet Systems}

\subsection{Time-Frequency Localization}

Perhaps the most useful property of wavelet system decomposition is the time-frequency localization which arises due to the translations and dilations. As in Section 2.2.1, let $\Omega(\Psi)=\left[\omega_{0}(\Psi), \omega_{1}(\Psi)\right]$ denote the frequency concentration of $\Psi$ and $R(\Psi)=$ $\left[t_{0}(\Psi), t_{1}(\Psi)\right]\left(t_{0} \geq 0\right)$ denote the time concentration of $\Psi$ (computed using the inverse Laplace transform). Then $\Psi$ is a function which is concentrated in the time frequency plane on the rectangle $\mathcal{Q}=\Omega(\Psi) \times R(\Psi)$ and each of the WS transfer functions are concentrated on rectangles

$\mathcal{Q}_{m n}=\left[a_{0}^{-m}\left(\omega_{0}(\Psi)+n b_{0}\right), a_{0}^{-m}\left(\omega_{1}(\Psi)+n b_{0}\right)\right] \times\left[a_{0}^{m} t_{0}(\Psi), a^{m} t_{1}(\Psi)\right]=\Omega\left(\Psi_{m, n}\right) \times R\left(\Psi_{m, n}\right)$.

Figure 5, shows the distribution of the rectangles $\mathcal{Q}_{m n}$ in the time-frequency plane. Thus the WS expansion (16) provides a decomposition of $\mathrm{H}^{2}\left(\Pi^{+}\right)$in which each term represents a finite-dimensional system which is localized in both time and frequency. Since the wavelets for $\mathrm{H}^{2}\left(\Pi^{+}\right)$are constructed in the frequency domain, the time and frequency axes are interchanged when compared to the analogous picture for wavelets constructed in the time-domain. A noteworthy feature of time-frequency localization properties of $\mathrm{H}^{2}\left(\Pi^{+}\right)$wavelet systems is that:

- Near $t=0$, while time localization is good, the frequency concentration of each wavelet system encompasses a large band of frequencies.

- For $t \gg 0$, frequency localization is very good so that it is possible to 'zoom' in on narrow frequency bands, whereas localization in time is poor. 

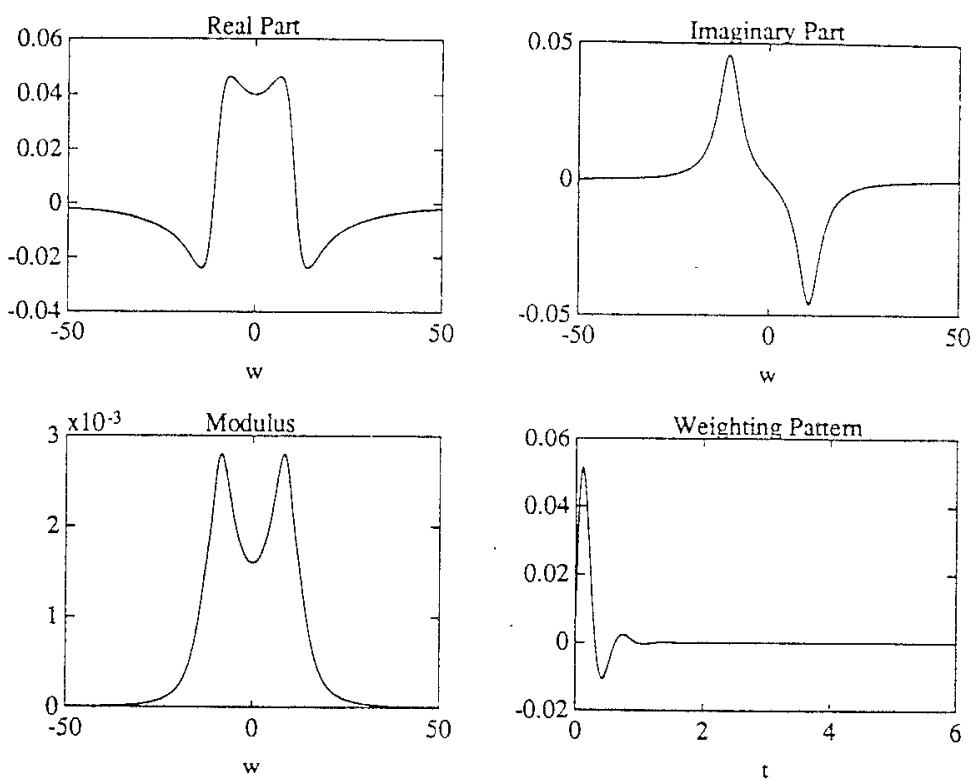

Figure 2: $\mathrm{H}^{2}\left(\Pi^{+}\right)$wavelet $\Psi$ for $\gamma=5, \xi=10$, and weighting pattern $\psi$.

\subsection{Poles and Zeros}

Given the poles and zeros of the analyzing wavelet $\Psi$ we would like to examine the manner in which the poles and zeros of the WS transfer functions are influenced by the translations and dilations. Assume first of all that $\Psi$ has no real axis poles and that $\Psi$ is a degree $N$ real-rational function in $\mathrm{H}^{2}\left(\Pi^{+}\right)$. Let $\left\{p_{k}, \bar{p}_{k}\right\}_{k=1}^{N / 2}$ be the set set of poles of $\Psi(s)$, and $\left\{z_{j}, \overline{z_{j}}\right\}_{j=1}^{J}$ be the set of zeros of $\Psi$. Since $\Psi$ is real-rational, $\Psi$ can be written as

$$
\Psi(s)=\frac{P(s)}{Q(s)}=\frac{\prod_{j}\left(s-z_{j}\right)\left(s-\overline{z_{j}}\right)}{\prod_{k}\left(s-p_{k}\right)\left(s-\overline{p_{k}}\right)}
$$

where, $P(s)$ and $Q(s)$ are relatively coprime. Thus,

$$
\begin{aligned}
\Psi_{m, n}(s) & =\frac{a_{0}^{m / 2} \prod_{j}\left(a_{0}^{m} s-z_{j}-i n b_{0}\right)\left(a_{0}^{m} s-\overline{z_{j}}-i n b_{0}\right)}{\prod_{k}\left(a_{0}^{m} s-p_{k}-i n b_{0}\right)\left(a_{0}^{m} s-\overline{p_{k}}-i n b_{0}\right)} \\
& =\frac{a_{0}^{m / 2} \prod_{j}\left(s-\beta_{j}(m, n)\right)\left(s-\gamma_{j}(m, n)\right)}{\prod_{k}\left(s-\eta_{k}(m, n)\right)\left(s-\nu_{k}(m, n)\right)}=\frac{P_{m, n}(s)}{Q_{m, n}(s)}
\end{aligned}
$$

where,

$$
\begin{array}{ll}
\beta_{k}(m, n)=a_{0}^{-m}\left(z_{j}+i n b_{0}\right) & \gamma_{k}(m, n)=a_{0}^{-m}\left(\overline{z_{j}}+i n b_{0}\right) \\
\eta_{k}(m, n)=a_{0}^{-m}\left(p_{k}+i n b_{0}\right) & \nu_{k}(m, n)=a_{0}^{-m}\left(\overline{p_{k}}+i n b_{0}\right)
\end{array}
$$

Note that $\eta_{k}(m,-n)=\overline{\nu_{k}}(m, n)$ and $\nu_{k}(m,-n)=\overline{\eta_{k}}(m, n)$ and $\beta_{k}(m,-n)=\overline{\gamma_{k}}(m, n)$ and $\gamma_{k}(m,-n)=\overline{\beta_{k}}(m, n)$. Therefore the poles of $G^{m, n}(s)=\alpha \Psi_{m, n}(s)+\bar{\alpha} \Psi_{m,-n}(s)$ are

$$
\left\{\eta_{k}(m, n), \overline{\eta_{k}}(m, n), v_{k}(m, n), \overline{\nu_{k}}(m, n)\right\} .
$$

Thus the effect of dilations and translations upon poles is, 


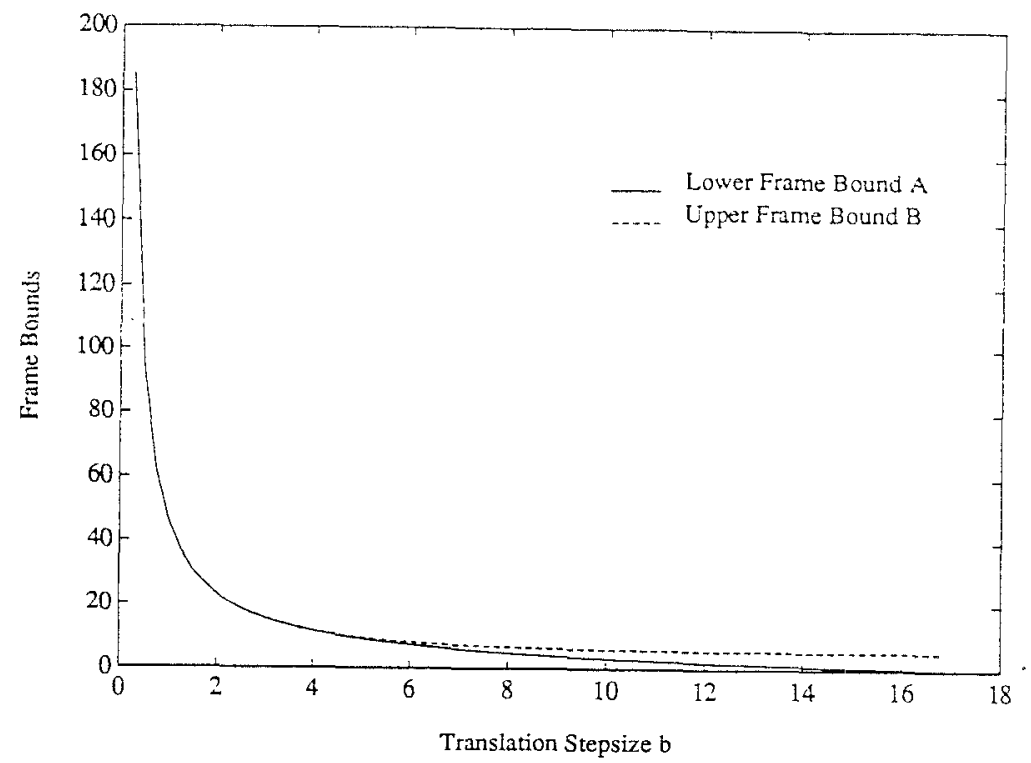

Figure 3: Estimates of frame bounds, using $\mathrm{H}^{2}\left(\Pi^{+}\right)$analyzing wavelet $\Psi$ of Example 1, with dilation stepsize $a=2$, as translation stepsize $b$ is varied. Solid curve represents the lower frame bound $A$ and the dashed curve represents the upper frame bound $B$.

- Dilations move the poles of $G^{m, n}$ radially away from and towards zero. As the dilation index $m$ increases, the poles move towards zero and as $m$ decreases the poles move away from zero. However, the poles remain in the closed left half-plane which is crucial since otherwise the $G^{m, n}$ would not remain in $\mathrm{H}^{2}\left(\Pi^{+}\right)$.

- The complex translations simply translate the poles along vertical lines in the left half-plane.

If we write $G^{m, n}(s)=N_{m, n}(s) / D_{m, n}(s)$, then the zeros of $G^{m, n}(s)$ are the roots of

$$
\begin{aligned}
N_{m, n}(s)= & \alpha P_{m, n}(s) D_{m,-n}(s)+\bar{\alpha} P_{m,-n}(s) D_{m, n}(s) \\
= & \alpha \prod_{j}\left(s-\beta_{j}(m, n)\right)\left(s-\gamma_{j}(m, n)\right) \prod_{k}\left(s-\overline{\eta_{k}}(m, n)\right)\left(s-\overline{\nu_{k}}(m, n)\right) \\
& +\bar{\alpha} \prod_{j}\left(s-\overline{\beta_{j}}(m, n)\right)\left(s-\overline{\gamma_{j}}(m, n)\right) \prod_{k}\left(s-\eta_{k}(m, n)\right)\left(s-\nu_{k}(m, n)\right)
\end{aligned}
$$

Without actually deriving expressions for the zeros of $G^{m, n}(s)$ we make the following observations:

- The zeros of $G^{m, n}(s)$ occur in complex conjugate pairs.

- For $n \neq 0$, if $G^{m, n}$ has no real-axis poles, then $N_{m, n}(s)$ and $D_{m, n}$ are coprime, and therefore $G^{m, n}(s)$ is a strictly proper rational function of order $2 N$.

- For $n=0$ pole-zero cancellation results in $G^{m, n}(s)$ of order $N$.

- If $l(n)$ is the number of poles of $G^{m, n}$ on the real axis then the order of $G^{m, n}(s)$ is $2 N-l(n)$. 


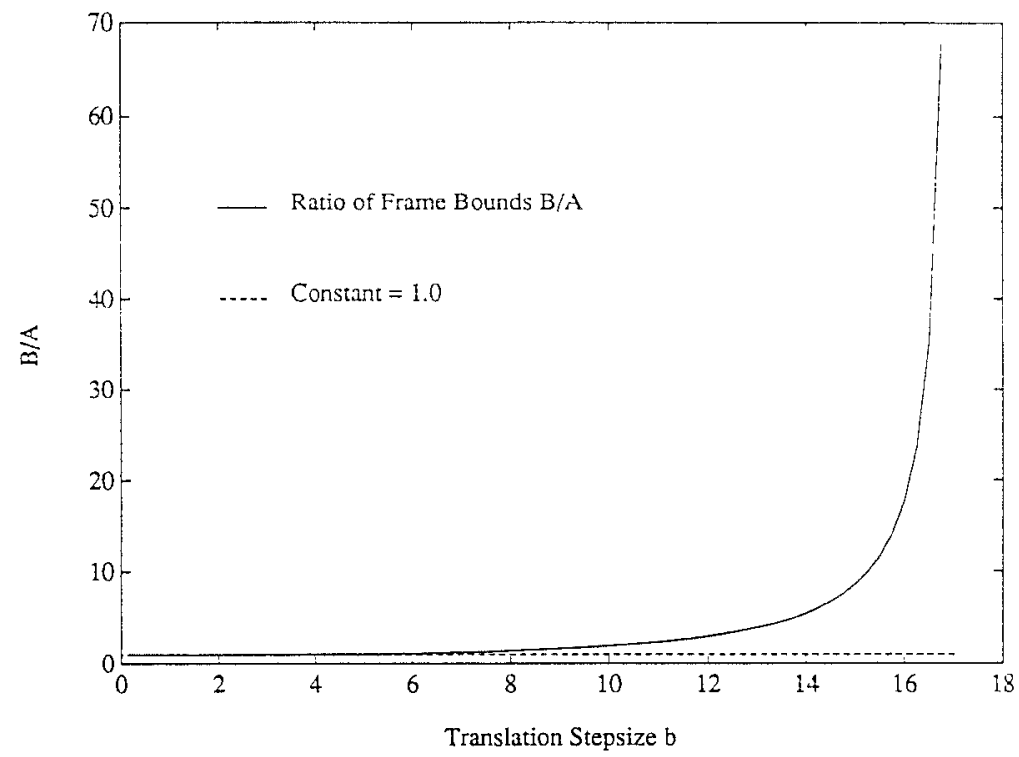

Figure 4: Ratio (B/A) of estimated frame bounds, using $\mathrm{H}^{2}\left(\Pi^{+}\right)$analyzing wavelet $\Psi$ of Example 1, with dilation stepsize $a=2$, as translation stepsize $b$ is varied. Solid curve represents $B / A$, and the.dashed line indicates the level where $B / A=1$.

\subsection{State Space Realizations of Wavelet System Transfer Func- tions}

Let $\psi(t)$ be the weighting pattern (i.e. inverse Laplace transform) corresponding to the real rational $\mathrm{H}^{2}\left(\Pi^{+}\right)$-wavelet $\Psi$ and define the triplet $(A, B, C)$ to be a minimal realization of $\Psi$. In this section we examine the question of realizations of the WS transfer function $G^{m, n}(s)=\alpha \Psi_{m, n}(s)+\alpha^{*} \Psi_{n,-n}(s)$. Let $\alpha_{R}$ and $\alpha_{I}$ denote respectively the real and imaginary parts of $\alpha$. Since $(A, B, C)$ is a realization of $\Psi$,

$$
\psi(t)=C \exp (A t) B
$$

Taking the inverse Laplace transform, the weighting pattern corresponding to $G^{m, n}(s)$ is,

$$
\begin{aligned}
g_{m, n}(t)= & \alpha a_{0}^{-m / 2} \psi\left(a_{0}^{-m} t\right) e^{i n a_{0}^{-m} b_{0} t}+\bar{\alpha} a_{0}^{-m / 2} \psi\left(a_{0}^{-m} t\right) e^{-i n a_{0}^{-m} b_{0} t} \\
= & a_{0}^{-m / 2} \psi\left(a_{0}^{-m} t\right) 2\left[\alpha_{R} \cos \left(n a_{0}^{-m} b_{0} t\right)-\alpha_{I} \sin \left(n a_{0}^{-m} b_{0} t\right)\right] \\
= & \alpha a_{0}^{-m / 2} C \exp \left(a_{0}^{-m} A t\right) B e^{i n a_{0}^{-m} b_{0} t}+\bar{\alpha} a_{0}^{-m / 2} C \exp \left(a_{0}^{-m} A t\right) B e^{-i n a_{0}^{-m} b_{0} t} \\
= & \alpha a_{0}^{-m / 2} C \exp \left(\left(a_{0}^{-m} A+i n a_{0}^{-m} b_{0} I\right) t\right) B \\
& \quad+\bar{\alpha} a_{0}^{-m / 2} C \exp \left(\left(a_{0}^{-m} A-i a_{0}^{-m} n b_{0} I\right) t\right) B .
\end{aligned}
$$

Equation (23) immediately provides a complex realization of $G^{m, n}(s)$ which is

$$
\begin{aligned}
A_{m n} & =\left[\begin{array}{cc}
a_{0}^{-m} A+i n a_{0}^{-m} b_{0} I & 0 \\
0 & a_{0}^{-m} A-i n a_{0}^{-m} b_{0} I
\end{array}\right] \\
B_{m n} & =\left[\begin{array}{ll}
B & B
\end{array}\right]^{T} \\
C_{m n} & =\left[\begin{array}{ll}
a_{0}^{-m / 2} \alpha C & a_{0}^{-m / 2} \bar{\alpha} C
\end{array}\right]
\end{aligned}
$$




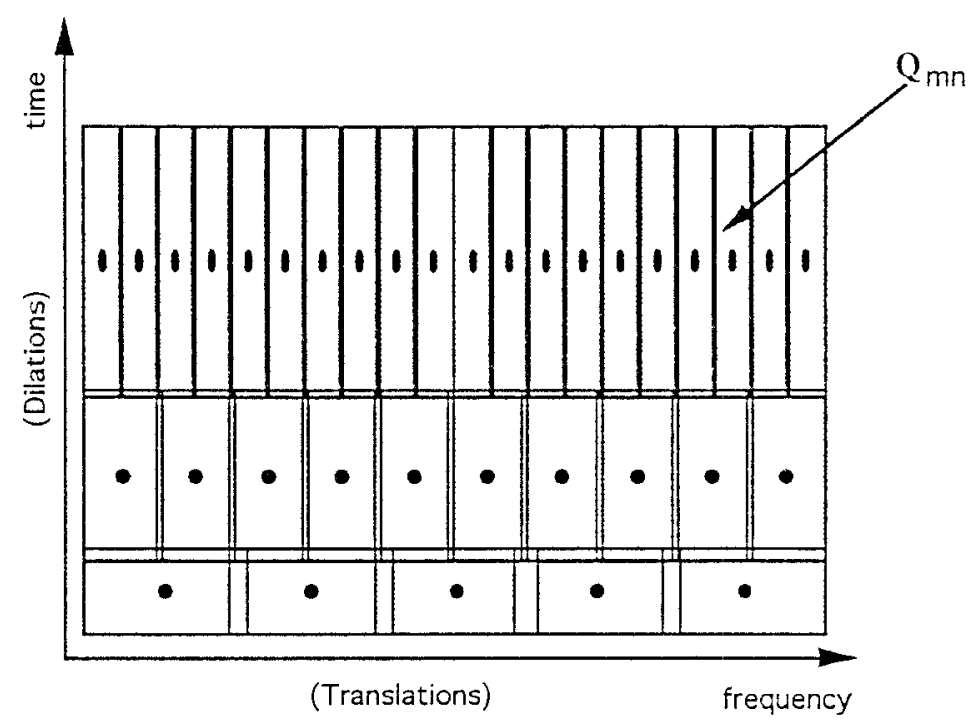

Figure 5: Time-frequency concentrations of wavelet systems.

However, since $\psi_{m, n}(t)$ is a real weighting pattern, we need a change of basis to give us a real realization. The differential equations corresponding to (26) are

$$
\begin{aligned}
& \dot{x_{1}}=\left(a_{0}^{-m} A+i n a_{0}^{-m} b_{0} I\right) x_{1}+B u \\
& \dot{x_{2}}=\left(a_{0}^{-m} A-i n a_{0}^{-m} b_{0} I\right) x_{2}+B u
\end{aligned}
$$

and the output map is given by

$$
y=a_{0}^{-m / 2} \alpha C x_{1}+a_{0}^{-m / 2} \bar{\alpha} C x_{2} .
$$

Let, $z_{1}=\left(x_{1}+x_{2}\right)$ and $z_{2}=i\left(x_{1}-x_{2}\right)$. Then we get

$$
\begin{aligned}
& \dot{z_{1}}=a_{0}^{-m} A z_{1}+n a_{0}^{-m} b_{0} z_{2}+2 B u \\
& \dot{z_{2}}=a_{0}^{-m} A z_{2}-n a_{0}^{-m} b_{0} z_{1} .
\end{aligned}
$$

Under this transformation the output map becomes

$$
\begin{aligned}
y & =a_{0}^{-m / 2}\left(\alpha_{R} C\left(x_{1}+x_{2}\right)+i \alpha_{I} C\left(x_{1}-x_{2}\right)\right) \\
& =a_{0}^{-m / 2}\left(\alpha_{R} C z_{1}+i \alpha_{I} C z_{2}\right) .
\end{aligned}
$$

From (29) and (29) we get the following real realization of $G^{m, n}(s)$,

$$
\begin{aligned}
A_{m n} & =\left[\begin{array}{cc}
a_{0}^{-m} A & n a_{0}^{-m} b_{0} I \\
-n a_{0}^{-m} b_{0} I & a_{0}^{-m} A
\end{array}\right], \quad n \neq 0 \\
B_{m n} & =\left[\begin{array}{ll}
2 B & 0
\end{array}\right]^{T}, \quad n \neq 0 \\
C_{m n} & =\left[\begin{array}{ll}
a_{0}^{-m / 2} \alpha_{R} C & a_{0}^{-m / 2} \alpha_{I} C
\end{array}\right], \quad n \neq 0 \\
\left(A_{m, 0}, B_{m, 0}, C_{m, 0}\right) & =\left(a_{0}^{-m} A, B, a_{0}^{-m / 2} \alpha C\right)
\end{aligned}
$$

It is interesting to note that, in this form (31-33), the dilations and translations which appear in the WS transfer function $G_{m, n}(s)$ affect the state space realization via dilations and translations as well, i.e.

$$
A_{m, n}=a_{0}^{-m}\left(\left[\begin{array}{cc}
A & 0 \\
0 & A
\end{array}\right]-n b_{0}\left[\begin{array}{cc}
0 & -I \\
I & 0
\end{array}\right]\right), \quad n \neq 0 .
$$




\subsubsection{Parallel Connections of WS Transfer Functions}

In constructing rational approximations (see Section 7), we will be considering parallel connections of WS transfer functions of the form,

$$
G_{\mathcal{J}}(s)=\sum_{(m, n) \in \mathcal{J}} G^{m, n}(s)
$$

where $\mathcal{J}$ is a finite index set. Given Equations (31-33) defining the realization $\left(A_{m, n}, B_{m, n}, C_{m, n}\right)$ of $G^{m, n}(s)$, a state space realization of $G_{\mathcal{J}}(s)$ is readily obtained.

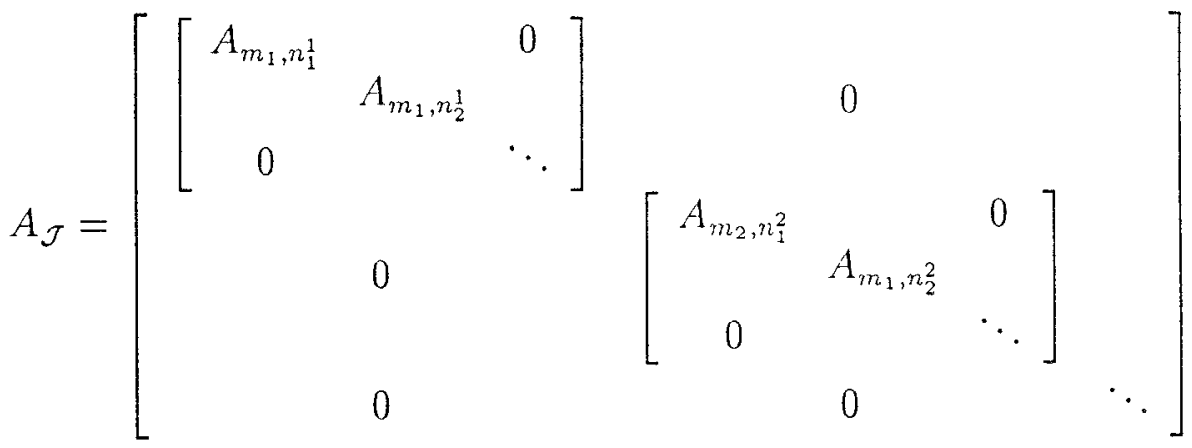

$$
\begin{aligned}
& B_{\mathcal{J}}=\left[B_{m_{1}, n_{1}^{1}}, B_{m_{1}, n_{2}^{1}}, \cdots\right]^{T} \\
& C_{\mathcal{J}}=\left[C_{m_{1}, n_{1}^{1}}, C_{m_{1}, n_{2}^{1}}, \cdots\right]
\end{aligned}
$$

\subsubsection{Minimality of State Space Realizations}

An obvious question that can be asked regarding the realization (36-38) of $G_{\mathcal{J}}(s)$ is whether such a realization is minimal in the sense of being both controllable and observable. We have the following result,

Theorem 5.1 Let $\Psi \in \mathrm{H}^{2}\left(\Pi^{+}\right)$be an admissible rational analyzing wavelet, let $\left\{p_{j}\right\}$ denote the poles of $\Psi$ and let $(A, B, C)$ be a minimal realization of $\Psi$ (of dimension $N$ ). Also let Jbe a finite, bounded index set. Then

(a) The realization (31-33) of $G^{m, n}(s)$ is minimal for all $(m, n) \in \mathcal{J}$ if and only if, for any $j=1, \ldots, N$, there do not exist any nonzero integers $k$, such that.

$$
\frac{\mathcal{I} m p_{j}}{b_{0}}=k
$$

(b) The realization (36-38) of $G_{\mathcal{J}}(s)$ is minimal if,

(1) $\left(A_{m, n}, B_{m, n}, C_{m, n}\right)$ as defined in $(31-39)$ is a minimal realization of $G^{m, n}(s)$ for all $(m, n) \in \mathcal{J}$, and

(2) For any $j, l=1, \ldots, N$, there do not exist any nonzero integers $k$, such that.

$$
\frac{1}{\log a_{0}} \log \left(\frac{-\Re e p_{j}}{-\Re e p_{l}}\right)=k
$$

Proof: (See Appendix B) 


\section{Computing the Wavelet System Decomposition}

In principle, given a frame $\left\{\Psi_{m, n}\right\}$ the coefficients of a WS decomposition of any $f \in \mathrm{H}^{2}\left(\Pi^{+}\right)$can be computed by first computing the dual frame $\left\{S^{-1} \Psi_{m, n}\right\}$ using the Neumann series expansion for the inverse frame operator $S^{-1}$ and then computing the inner products of $f$ with elements of the dual frame. However in practice, this may prove to be an extremely cumbersome computation, especially in cases where the frame is not close to being a tight frame $(B / A=1)$. Expansions with respect to frames are in general not unique due to the lack of linear independence. It can be seen from Theorem 2.1 that among all possible expansion coefficients for a given function $f$, the coefficient sequence $c=\left\{\left\langle f, S^{-1} \Psi_{m, n}\right\rangle\right\}$ is optimal in the sense of minimum $\ell^{2}\left(\mathbb{Z}^{2}\right)$ norm. That is if $\left\{\alpha_{m, n}\right\}$ is such that

$$
f=\sum_{m} \sum_{n} \alpha_{m, n} \Psi_{m, n}
$$

then,

$$
\sum_{m} \sum_{n}\left|\left\langle f, S^{-1} \Psi_{m, n}\right\rangle\right|^{2} \leq \sum_{m} \sum_{n}\left|\alpha_{m, n}\right|^{2} .
$$

For WS approximations using a finite number of terms, the problem of computing coefficients may be posed as a least squares approximation problem. Among all possible sequences of approximation coefficients, the minimum $\ell^{2}$ norm coefficient sequence can be obtained by casting the least squares problem into the form of a system of linear equations and then utilizing the generalized inverse $P^{\dagger}=\left(P^{*} P\right)^{-1} P^{*}$ to solve for the coefficients.

\subsection{Example: WS Decomposition}

Example 1: Heat Equation with Dirichlet Boundary Control

Consider a system defined by the partial differential equation,

$$
\left\{\begin{array}{l}
\frac{\partial z}{\partial t}=\frac{\partial^{2} z}{\partial x^{2}} ; \quad z(0)=0, z(1)=u(t) \\
y(t)=z\left(x_{0}, t\right)
\end{array}\right.
$$

This system has transfer function [4]

$$
G_{1}(s)=\frac{\sinh \sqrt{s} x_{0}}{\sinh \sqrt{s}} .
$$

By assuming lowpass characteristics of the sensor, we can write the overall transfer function as ,

$$
G(s)=\frac{1}{s+\pi} \frac{\sinh \sqrt{s} x_{0}}{\sinh \sqrt{s}} .
$$

A decomposition of $\mathrm{G}(\mathrm{s})$ was computed using 12 dilation levels $(m=-5, \ldots, 6)$ and up to 33 translations at each dilation level. The results of this decomposition are shown in Figures 6-8 which are different representations of the magnitude of the wavelet system expansion coefficients. Along the dilation axis, zero corresponds to the lowest dilation level $(m=-5)$, and along the translation axis zero corresponds to $n=0$. Figures 6 and 
7 are 3D and contour plots respectively of the coefficient magnitudes and Figure 8 is a density plot in which each rectangle is shaded according to the corresponding coefficient magnitude. The key feature in this decomposition, which is probably most obvious

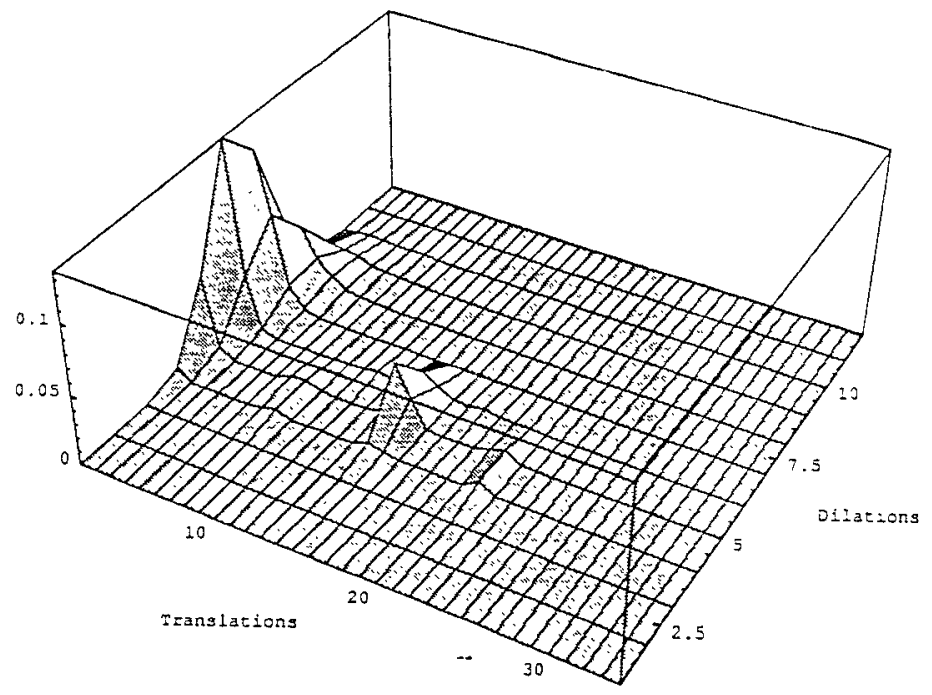

Figure 6: Wavelet system decomposition of heat equation transfer function - 3D plot of magnitude of expansion coefficients

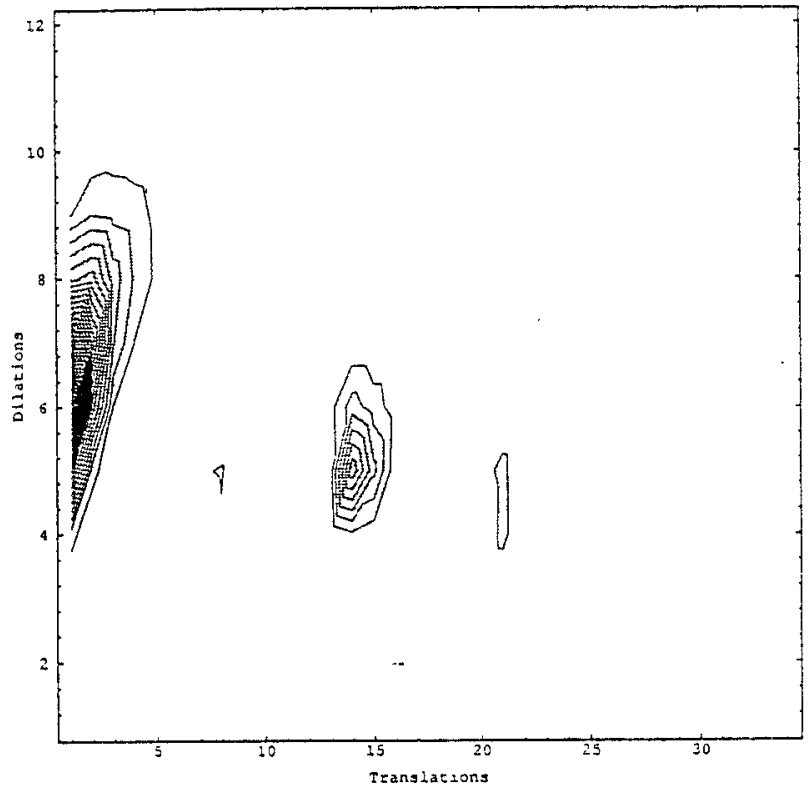

Figure 7: Wavelet system decomposition of heat equation transfer function - contour plot of magnitude of expansion coefficients

in the density plot (Figure 8) is that the magnitudes of the coefficients are very well concentrated. This feature, which is due to the time-frequency localization properties of affine wavelets, permits us to pick a finite number of 'significant' terms in the wavelet system decomposition. In doing so, a finite-dimensional approximation to the original transfer function is obtained. Using the results of Section 5.3, it is also possible to immediately write down a minimal state space realization of the approximating system. 


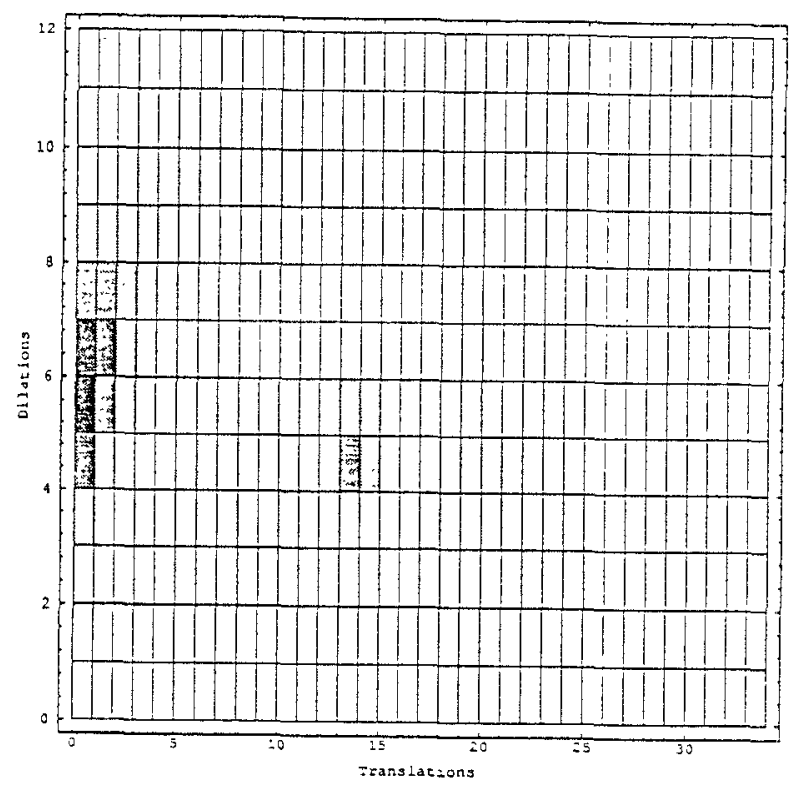

Figure 8: Wavelet system decomposition of heat equation transfer function - density plot of magnitude of expansion coefficients

\section{Rational WS Approximations to Nonrational $\mathrm{H} 2(\Pi+)$ Transfer Functions}

Wavelet system decompositions as in (16) provide a means of representing nonrational transfer functions in $\mathrm{H}^{2}\left(\Pi^{+}\right)$as infinite sums of rational transfer functions ${ }^{1}$. As mentioned earlier, our primary objective in deriving such a decomposition is to devise a systematic method of constructing rational approximations. What is required now is a mechanism which allows for judicious selection of a finite number of terms from the expansion in (16). For this purpose we utilize the localization properties afforded us by affine wavelets.

For a significant class of transfer functions arising from physical systems, the WS decomposition will result in a reasonably compact representation in the sense of well localized and rapidly decaying coefficients. This phenomenon can be explained on the basis of the time-frequency localization properties of the WS transfer functions and the observation that $\mathrm{H}^{2}\left(\Pi^{+}\right)$transfer functions arising from physical systems are often well localized in time-frequency as well. It is possible to devise a variety of schemes, each relying on time-frequency localization properties, for the selection of terms in a WS decomposition for use in a finite-dimensional approximation.

One possible choice for the selection of a finite-dimensional WS approximation can be based upon knowledge of the time-frequency concentration of the (nonrational) transfer function which is to be approximated. For example given that the time-frequency concentration of a transfer function $f \in \mathrm{H}^{2}\left(\Pi^{+}\right)$is $\mathcal{Q}(f)$ we can select a subset of the wavelet system transfer functions based upon the size of $\mathcal{Q}(f) \cap \mathcal{Q}_{m, n}$, where $\mathcal{Q}_{m, n}$ is the concentration of the WS transfer function $G^{m, n}(s)$. Daubechies in [6] provides bounds for the error of such approximations in terms of the energy of $f$ outside $\mathcal{Q}(f)$. A second

\footnotetext{
${ }^{1}$ Note that causality is preserved due to the property described by Equation (9)
} 
selection scheme could consist of first computing the wavelet expansion coefficients for a large number of terms and then simply discarding those terms with 'small' coefficients. This second method is similar to those used to achieve signal or image compression via wavelet decompositions (c.f. $[3,18]$ ). One such selection criterion, can be based upon the $\ell^{2}$ norm of the coefficients. Let, us assume that we have computed the WS decomposition of a transfer function $G_{T}$ and let $\alpha_{m . n}=\left\langle G, S^{-1} \Psi_{m, n}\right\rangle$ denote the coefficients. We choose the $K$ largest coefficients, (whose indices we store in an index set $\mathcal{J}$ ), where $K=\#(\mathcal{J})$ is the smallest integer such that,

$$
\frac{\sum_{(m, n) \in \mathcal{J}}\left|\alpha_{m, n}\right|^{2}}{\sum_{(m, n)}\left|\alpha_{m, n}\right|^{2}} \geq(1-\delta)
$$

where $0 \leq \delta<1$ is some predetermined tolerance. We now define a rational approximation, $G_{\mathcal{J}}$, to $G$ as, $G_{\mathcal{J}}(s)=\sum_{(m, n) \in \mathcal{J}} G^{m, n}(s)$. Note that the results of Section 5.3 immediately provide a minimal state space realization of $G_{\mathcal{J}}$.

\subsection{Approximation Error Bounds}

The following lemma provides a bound on the approximation error using the scheme just described.

Lemma 7.1 Let $\left\{x_{n}\right\}_{n} \in \mathbb{Z}$ be a frame for a Hilbert space $\mathcal{H}$, with frame bounds $A$ and B. Assume $\left\|x_{n}\right\|=1$, for all $n \in \mathbb{Z}$. For any $f \in \mathcal{H}$, define an approximation $\tilde{f}$ to $f$ $b y$,

$$
\tilde{f}=\sum_{n \in \mathcal{J}}\left\langle f, S^{-1} x_{n}\right\rangle x_{n},
$$

where $J$ is an index set, chosen to satisfy

$$
\sum_{n \in \mathcal{J}}\left|\left\langle f, S^{-1} x_{n}\right\rangle\right|^{2} \geq(1-\delta) \sum_{n \in \mathbf{Z}}\left|\left\langle f, S^{-1} x_{n}\right\rangle\right|^{2}
$$

for $0 \leq \delta<1$. Then,

$$
\|f-\widetilde{f}\|^{2} \leq \delta \frac{B}{A}\|f\|^{2}
$$

Proof: By the frame condition,

$$
B^{-1}\|f-\tilde{f}\|^{2} \leq \sum_{k \in \mathbf{Z}}\left|\left\langle f-\tilde{f}, S^{-1} x_{k}\right\rangle\right|^{2} \leq A^{-1}\|f-\tilde{f}\|^{2} .
$$

Since, $f-\tilde{f}=\sum_{n \notin \mathcal{J}}\left\langle f, S^{-1} x_{n}\right\rangle x_{n}$, we have two coefficients sequences representing the expansion of $f-\widetilde{f}$ with respect to the frame $\left\{x_{n}\right\}$. Therefore by Theorem 2.1,

$$
\sum_{n \notin \mathcal{J}}\left|\left\langle f, S^{-1} x_{n}\right\rangle\right|^{2} \geq \sum_{k \in \mathbf{Z}}\left|\left\langle f-\widetilde{f}, S^{-1} x_{k}\right\rangle\right|^{2} .
$$


Therefore,

$$
\begin{aligned}
B^{-1}\|f-\tilde{f}\|^{2} & \leq \sum_{k \in \mathbf{Z}}\left|\left\langle f-\tilde{f}, S^{-1} x_{k}\right\rangle\right|^{2} \\
& \leq \sum_{n \notin \mathcal{J}}\left|\left\langle f, S^{-1} x_{n}\right\rangle\right|^{2} \\
& \leq \delta \sum_{k \in \mathbf{Z}}\left|\left\langle f, S^{-1} x_{k}\right\rangle\right|^{2} \\
& \leq \delta A^{-1}\|f\|^{2}
\end{aligned}
$$

from which the result follows.

Remark: The error bound in Lemma 7.1 is established in the general setting of frames in Hilbert spaces. When applied to the specific case of affine wavelet frames, the bound may prove to be quite conservative. This is because time-frequency localization properties of affine wavelets are not exploited in the lemma.

\subsection{Example: Rational WS Approximation}

As an example, consider the WS decomposition of the heat equation transfer function in Section 6.1. Letting $\delta=0.4$ the above described scheme results in the selection of 7 terms, with a corresponding normalized $L^{2}$ approximation error of 0.109 , and an approximating system of dimension 22 .

\section{Conclusions and Discussion}

In this paper we have introduced a new decomposition (which we call a wavelet system decomposition ) of $\mathrm{H}^{2}\left(\Pi^{+}\right)$. WS decompositions provide a representation of $\mathrm{H}^{2}\left(\Pi^{+}\right)$ as an infinite sum of rational functions of bounded degree. Construction of the WS representation is based on an appropriate grouping of terms in an affine wavelet frame decomposition of $\mathrm{H}^{2}\left(\Pi^{+}\right)$where the analyzing wavelet is rational. For a rational analyzing wavelet of degree $N$ the terms in a WS expansion are either of degree $N$ or $2 N$.

WS decomposition naturally leads to methods for constructing rational approximations to nonrational transfer functions in $\mathrm{H}^{2}\left(\Pi^{+}\right)$. Criteria for selecting a finite number of terms from the infinite wavelet system expansion rely on compactness of representation which arises due to time-frequency localization properties of the wavelet systems. Much work remains to be done to understand the approach of the present paper against the background of prior work on $\mathrm{L}^{2}$ and $\mathrm{H}^{\infty}$ approximation theory (c.f. $[11,10,12,1]$ ).

A point should be made regarding our motivation for constructing affine frames in the frequency (Laplace) domain as opposed to directly decomposing weighting patterns via affine wavelets in the time domain. First of all, our approach naturally preserves causality of the approximating system since each term in the WS expansion is in $\mathrm{H}^{2}\left(\Pi^{+}\right)$ and therefore corresponds to the transfer function of a causal system. If one were to 
use affine wavelets in the time domain, special 'tricks' need to be applied to preserve causality since (even in the case of compactly supported wavelets), translations would eventually result in a noncausal wavelet. Secondly, there would be no mechanism for retaining rationality of the Laplace transforms of the individual wavelets, since translations (delays) prevent this.

As a concluding remark, we should mention that the following result has recently come to our attention [16]

Theorem 8.1 There exist no orthonormal bases of wavelets for $\mathrm{H}^{2}\left(\Pi^{+}\right)$generated via the framework of multiresolution analyses, with an analyzing wavelet $\psi$ such that $\widehat{\psi}$ is continuous and,

$$
|\widehat{\psi(\omega)}| \leq C|\omega|^{-\alpha} \text { for any } \alpha>1 / 2 \text {. }
$$

In particular this rules out smooth and well localized orthonormal bases of rational wavelets for $\mathrm{H}^{2}\left(\Pi^{+}\right)$. Although it has not been demonstrated that multiresolution analyses are the only means of constructing wavelet orthonormal bases, all known examples of orthonormal wavelet bases can be associated with multiresolution analyses. It remains an open problem to construct an orthonormal wavelet basis (even for $L^{2}(\mathbb{R})$ ) which does not arise in this way. The above theorem suggests that if one wishes to use rational analyzing wavelets, it is perhaps necessary to consider the more general setting of frames (including Riesz bases).

\section{References}

[1] L. Baratchart. Recent and new results in $\mathrm{L}^{2}$ rational approximation. In R. F. Curtain, editor, Modelling, Robustness and Sensitivity Reduction in Control Systems. Springer-Verlag, Berlin, 1987.

[2] R. Brockett and P. S. Krishnaprasad. A scaling theory for linear systems. IEEE Transactions on Automatic Control, AC-25(2):197-207, 1980.

[3] R. R. Coifman, Y. Meyer, S. Quake, and M. V. Wickerhauser. Signal processing and compression with wavelet packets. preprint, Yale University, April 1990.

[4] R. F. Curtain. A synthesis of time and frequency domain methods for the control of infinite-dimensional systems: A system theoretic approach. Preprint, Univ. of Groningen, The Netherlands, September 1988.

[5] I. Daubechies. Orthonormal bases of compactly supported wavelets. Communications on Pure and Applied Mathematics, 41:909-996, 1988.

[6] I. Daubechies. The wavelet transform, time-frequency localization and signal analysis. IEEE Transactions on Information Theory, 36(5), September 1990.

[7] R. J. Duffin and A. C. Schaeffer. A class of nonharmonic fourier series. Trans. Amer. Math. Soc., 72:341-366, 1952.

[8] P. L. Duren. Theory of $H^{p}$ Spaces, volume 38. Academic Press, New York, 1970. 
[9] J. B. Garnett. Bounded Analytic Functions, volume 96. Academic Press, New York, 1981.

[10] K. Glover, J. Lam, and J. R. Partington. Rational approximation of a class of infinite-dimensional systems I: Singular values of Hankel operators. Mathematics of Control, Signals, and System.s, 3(4):325-344, 1990.

[11] K. Glover, J. Lam, and J. R. Partington. Rational approximation of a class of infinite-dimensional systems: The $\mathrm{L}^{2}$ case. In P. Nevai and A. Pinkus, editors, Progress in Approximation Theory. Academic Press, San Diego, 1991.

[12] K. Glover, J. Lam, and J. R. Partington. Rational approximation of a class of infinite-dimensional systems II: Optimal convergence rates of $\mathrm{L}_{\infty}$ approximants. Mathematics of Control, Signals, and Systems, 4(3):23:3-246, 1991.

[13] A. Grossmann and J. Morlet. Decomposition of hardy functions into square integrable wavelets of constant shape. SIAM Journal of Mathematical Analysis, 15(4):723-736, July 1984.

[14] C. E. Heil and D. F. Walnut. Continuous and discrete wavelet transforms. SIAM Review, 31(4):628-666, December 1989.

[15] K. Hoffman. Banach Spaces of Analytic Functions. Dover Publications, New York, 1988.

[16] S. Jaffard and Ph. Laurencot. Orthonormal wavelets, analysis of operators, and applications to numerical analysis. In C. K. Chui, editor, Wavelets: A Tutorial in Theory and Applications. Academic Press, San Diego, 1992.

[17] Y. C. Pati and P. S. Krishnaprasad. Analysis and synthesis of feedforward neural networks using discrete affine wavelet transforms. IEEE Transactions on Neural Networks, 1992. In Press. (Also Univ. of Md., Systems Research Center Technical Report SRC-TR-90-44, 28 pages.).

[18] M. V. Wickerhauser. Acoustic signal compression with wave packets. preprint, Yale University, 1989.

\section{Appendix A}

\section{Proof of Proposition 4.2}

Without loss of generality we can consider the case $m=0$. Therefore we need to check that for $b \in \mathbb{R}$,

$$
\int_{\mathbb{R}} f(\omega) g(\omega+b) d \omega-\overline{\left(\int_{\mathbb{R}} f(\omega) g(\omega-b) d \omega\right)}=0 .
$$

Consider the real and imaginary parts of the l.h.s. of Equation (A.43) separately. 
Real part Taking the real part of Equation (A.4:3),

$$
\begin{aligned}
& \Re e\left(\int_{\mathbb{R}} f(\omega) g(\omega+b) d \omega-\overline{\left(\int_{\mathbb{R}} f(\omega) g(\omega-b) d \omega\right)}\right) \\
= & \Re e\left(\int_{\mathbb{R}} f(\omega) g(\omega+b) d \omega-\int_{\mathbb{R}} f(\omega) g(\omega-b) d \omega\right) \\
= & \Re e\left(\int_{-\infty}^{0} f(\omega)(g(\omega+b)-g(\omega-b)) d \omega+\int_{0}^{\infty} f(\omega)(g(\omega+b)-g(\omega-b)) d \omega\right) \\
= & \Re e\left(-\int_{\infty}^{0} f(-\omega)(g(-\omega+b)-g(-\omega-b)) d \omega+\int_{0}^{\infty} f(\omega)(g(\omega+b)-g(\omega-b)) d \omega\right) \\
= & \Re e\left(\int_{0}^{\infty} f(-\omega)(g(-(\omega-b))-g(-(\omega+b))) d \omega+\int_{0}^{\infty} f(\omega)(g(\omega+b)-g(\omega-b)) d \omega\right) \\
= & \Re e(\int_{0}^{\infty} \underbrace{f(\omega)(\bar{g}(\omega-b)-\bar{g}(\omega+b))}_{H(\omega)} d \omega+\int_{0}^{\infty} \underbrace{f(\omega)(g(\omega+b)-g(\omega-b))}_{-\bar{H}(\omega)} d \omega) \\
= & \Re e\left(\int_{0}^{\infty}[H(\omega)-\bar{H}(\omega)] d \omega\right)=0
\end{aligned}
$$

\section{Imaginary part}

$$
\begin{aligned}
& \operatorname{Im}\left(\int_{\mathbb{R}} f(\omega) g(\omega+b) d \omega-\overline{\left(\int_{\mathbb{R}} f(\omega) g(\omega-b) d \omega\right)}\right) \\
= & \operatorname{Im}\left(\int_{\mathbb{R}} f(\omega) g(\omega+b) d \omega+\int_{\mathbb{R}} f(\omega) g(\omega-b) d \omega\right) \\
= & \operatorname{Im}\left(\int_{-\infty}^{0} f(\omega)(g(\omega+b)+g(\omega-b)) d \omega+\int_{0}^{\infty} f(\omega)(g(\omega+b)+g(\omega-b)) d \omega\right) \\
= & \operatorname{Im}\left(-\int_{\infty}^{0} f(-\omega)(g(-\omega+b)+g(-\omega-b)) d \omega+\int_{0}^{\infty} f(\omega)(g(\omega+b)+g(\omega-b)) d \omega\right) \\
= & \operatorname{Im}\left(\int_{0}^{\infty} f(-\omega)(g(-(\omega-b))+g(-(\omega+b))) d \omega+\int_{0}^{\infty} f(\omega)(g(\omega+b)+g(\omega-b)) d \omega\right) \\
= & \operatorname{Im}(\int_{0}^{\infty} \underbrace{\bar{f}(\omega)(\bar{g}(\omega-b)+\bar{g}(\omega+b))}_{H(\omega)} d \omega+\int_{0}^{\infty} \underbrace{f(\omega)(g(\omega+b)+g(\omega-b))}_{\bar{H}(\omega)} d \omega) \\
= & \operatorname{Im}\left(\int_{0}^{\infty}[H(\omega)+\bar{H}(\omega)] d \omega\right)=0
\end{aligned}
$$

Which proves the proposition

\section{Appendix B}

\section{Proof of Theorem 5.1}


We outline here the proof of Theorem 5.1.

Proof of part (a): Since (31-33) is a $2 N$-dimensional realization of $G^{m, n}(s)$, we need only prove that the rational function $G^{m, n}(s)$ is of order $2 N$ for all $n \in \mathbb{Z}, n \neq 0$ if and only if (39) holds.

The poles of $G^{m, n}(s)($ see $(21))$ are,

$$
\left\{\eta_{k}(m, n), \overline{\eta_{k}}(m, n), \nu_{k}(m, n), \overline{\nu_{k}}(m, n)\right\}
$$

where $\eta_{k}(m, n)$ and $\nu_{k}(m, n)$ are defined by (20). Assuming (39) holds, $\eta_{k}(m, n)$ and $\nu_{k}(m, n)$ have nonzero imaginary parts for all $m, n \in \mathbb{Z}$. Therefore, at $s=\eta_{k}(m, n)$ for example, the term,

$$
\prod_{k}\left(s-\overline{\eta_{k}}(m, n)\right)\left(s-\overline{\nu_{k}}(m, n)\right)
$$

which appears in the numerator polynomial $N_{n, n}(s)$ of $G^{m, n}$ will be nonzero. Furthermore, the assumption that $\Psi$ is of order $N$, implies that $p_{j} \neq z_{k}$ for all $z, k$. Therefore at $s=\eta_{k}(m, n)$ the remaining (nonzero) term in the numerator of $G^{m, n}$ will be,

$$
N_{m, n}\left(\eta_{k}(m, n)\right)=\alpha \prod_{j}\left(s-\beta_{j}(m, n)\right)\left(s-\gamma_{j}(m, n)\right) \prod_{k}\left(s-\overline{\eta_{k}}(m, n)\right)\left(s-\overline{\nu_{k}}(m, n)\right),
$$

which shows that $G^{m, n}$ has no zeros at $\eta_{k}(m, n)$. Exactly the same argument can be used to show that there is no cancellation with zeros for the remaining poles. Thus $G^{m, n}$ is of order $2 N$.

The converse is readily proved using a similar pole-zero cancellation argument.

Proof of part (b): By hypothesis, $\left(A_{m, n}, B_{m, n}, C_{m, n}\right)$ is a minimal realization of $G^{m, n}(s)=N_{m, n}(s) / D_{m, n}(s)$ for all $(m, n) \in \mathcal{J}$. Therefore $N_{m, n}$ and $D_{m, n}$ are coprime polynomials. Consider the parallel combination of two WS transfer functions.

$$
G_{\mathcal{J}}(s)=G_{m_{1}, n_{1}}(s)+G_{m_{2}, n_{2}}(s),
$$

where $\mathcal{J} \quad=\left\{\left(m_{1}, n_{1}\right),\left(m_{2}, n_{2}\right)\right\}$. In this case $\left(N_{m_{1}, n_{1}}(s), D_{m_{2}, n_{2}}(s)\right)$ and $\left(N_{m_{2}, n_{2}}(s) D_{m_{1}, n_{1}}(s)\right)$, are relatively coprime pairs. Rewriting (B.44),

$$
\begin{aligned}
G_{\mathcal{J}}(s) & =\frac{N_{\mathcal{J}}(s)}{D_{\mathcal{J}}(s)} \\
& =\frac{N_{m_{1}, n_{1}}(s) D_{m_{2}, n_{2}}(s)+N_{m_{2}, n_{2}}(s) D_{m_{1}, n_{1}}(s)}{D_{m_{1}, n_{1}}(s) D_{m_{2}, n_{2}}(s)}
\end{aligned}
$$

Therefore $N_{\mathcal{J}}$ and $D_{\mathcal{J}}(s)$ will be relatively coprime if and only if $D_{m_{1}, n_{1}}$ and $D_{m_{2}, n_{2}}(s)$ are relatively coprime. The roots of $D_{m_{1}, n_{1}}$ and $D_{m_{2}, n_{2}}(s)$ are given by (21). Thus $D_{m_{1}, n_{1}}$ and $D_{m_{2}, n_{2}}(s)$ will have a common root if and only if,

$$
a_{0}^{-m_{1}}\left(p_{l}+i n_{1} b_{0}\right)=a_{0}^{-m_{2}}\left(p_{j} \pm i n_{2} b_{0}\right),
$$


for some $l$ and $j$. Solving for $n_{1}$,

$$
\begin{aligned}
n_{1}= & \frac{-i}{b_{0}}\left(a_{0}^{m_{1}-m_{2}} p_{j}-p_{l}\right) \pm a_{0}^{m_{1}-m_{2}} n_{2} \\
= & \frac{-i}{b_{0}}\left(a_{0}^{m_{1}-m_{2}}\left(\Re e p_{j}+i \operatorname{Im} p_{j}\right)-\left(\Re e p_{l}+i \operatorname{Im} p_{l}\right)\right) \pm a_{0}^{m_{1}-m_{2}} n_{2} \\
= & \left(\frac{1}{b_{0}}\left(a_{0}^{m_{1}-m_{2}} \mathcal{I} m p_{j}-\mathcal{I} m p_{l}\right) \pm a_{0}^{m_{1}-m_{2}} n_{2}\right) \\
& -\frac{i}{b_{0}}\left(a_{0}^{m_{1}-m_{2}} \Re e p_{j}-\Re e p_{l}\right)
\end{aligned}
$$

However, by hypothesis (40) there can be no integer solution $n_{1}$ to (B.45) since the imaginary part of (B.45) will always be nonzero. The above argument for two WS transfer functions is easily extended to any finite number of WS transfer functions in parallel.

\section{Appendix $\mathrm{C}$}

\section{Computation of Frame Parameters}

Given an admissible analyzing wavelet $g$, the following theorem of Daubechies is useful in determining dilation step-size $a$ and translation step-size $b$ for which $(g, a, b)$ generates an affine frame for $L^{2}(\mathbb{R})$.

Theorem C.1 (Daubechies [6]) Let $g \in \mathrm{L}^{2}(\mathbb{R})$ and $a>1$ be such that:

$$
m(g ; a)=\operatorname{ess} \inf _{|\omega| \in[1, a]} \sum_{n}\left|\widehat{g}\left(a^{n} \cdot \omega\right)\right|^{2}>0
$$

$$
M(g ; a)=\operatorname{ess} \sup _{|\omega| \in[1, a]} \sum_{n}\left|\widehat{g}\left(a^{n} \omega\right)\right|^{2}<\infty
$$

$$
\lim _{b \rightarrow 0} 2 \sum_{k=1}^{\infty} \beta(2 \pi k / b)^{1 / 2} \beta(-2 \pi k / b)^{1 / 2}=0
$$

where

$$
\beta(s)=\operatorname{ess} \sup _{|\omega| \in[1, a]} \sum_{n}\left|\widehat{g}\left(a^{n} \omega\right) \| \widehat{g}\left(a^{n} \omega-s\right)\right| .
$$

Then there exists $B_{c}>0$ such that $(g, a, b)$ generates an affine frame for each $0<b<B_{c}$.

Proof of the following corollary, can also be found in [6]. 
Corollary C.1 If $g \in \mathrm{L}^{2}(\mathbb{R})$ and $a>1$ satisfy the hypotheses of Theorem C.1 then,

$$
B_{c} \geq b_{c}=\inf \left\{b \mid m(g ; a)-2 \sum_{k=1}^{\infty} \beta(2 \pi k / b)^{1 / 2} \beta(-2 \pi k / b)^{1 / 2} \leq 0\right\}
$$

and for $0<b<b_{c}$, the frame bounds $A$ and $B$ can be estimated as,

$$
\begin{aligned}
& A \geq b^{-1}\left(m(g ; a)-2 \sum_{k=1}^{\infty} \beta(2 \pi k / b)^{1 / 2} \beta(-2 \pi k / b)^{1 / 2}\right) \\
& B \leq b^{-1}\left(M(g ; a)+2 \sum_{k=1}^{\infty} \beta(2 \pi k / b)^{1 / 2} \beta(-2 \pi k / b)^{1 / 2}\right)
\end{aligned}
$$


